\title{
Optimization Methodology for Efficient LLC Resonant Converter with Power Factor Correction Circuit
}

\author{
Ruiyang Yu*, Godwin Kwun Yuan Ho*, Bryan Man Hay Pong* \\ ${ }^{*}$ The University of Hong Kong,Department of Electrical and Electronic Engineering, yry721@eee.hku.hk; mhp@eee.hku.hk
}

Keywords: Efficiency, LLC series resonant converter, Optimization, Power factor correction.

\begin{abstract}
High efficiency is required in power conversion circuits. A systematic optimization procedure is presented in this paper to optimize LLC series resonant converter efficiency where the effect of LLC input voltage variation cased by power factor correction circuit is considered. A mode solver technique is proposed to handle LLC converter steady-state solutions under different input voltage conditions. Loss models are provided to calculate total component losses using the current and voltage information derived from the mode solver. A prototype $300 \mathrm{~W} 12 \mathrm{~V}$ output LLC converter is built using the optimization results. Experimental results are presented.
\end{abstract}

\section{Introduction}

Computer-aided-design (CAD) and optimization is an important art widely used in many applications. Design optimization of PWM converters were explored by researchers in the past few decades [1] [2] [3]. Nowadays, high efficiency is required for off-line power supplies.

LLC series resonant converter is emerging for high efficiency requirement. So far, there is not much work on the optimization of LLC converter. The optimization of LLC converter is more difficult than conventional PWM converters. It is because of the following reasons. First, there are multiple modes of operations; each mode has different resonant characteristics. Second, the nonlinear behavior of LLC converter does not have closed-form solutions. Third, LLC resonant converter is regulated by frequency. The input voltage of LLC has line frequency voltage ripple cased by the front end power factor correction circuit (PFC). It is difficult to calculate the accurate frequency that regulates the output voltage.

This research work proposes an optimization procedure that solves the above difficulties. The optimization procedure handles efficiency optimization problems where the LLC converter input ripple voltage from the PFC is considered. A mode solver technique is proposed to handle LLC converter steady-state solutions at different input voltages. The proposed mode solver utilizes numerical non-linear programming techniques to solve LLC state equations and determine operation mode. Loss models are provided to calculate total component losses using the current and voltage information derived from the mode solver. The calculated efficiency serves as the objective function to optimize converter efficiency. A prototype $300 \mathrm{~W} 12 \mathrm{~V}$ output voltage LLC resonant converter is built using the optimization results.

\section{Converter Models}

\subsection{PFC output voltage ripple}

Fig. 1 shows a typical two stage AC/DC DC/DC configuration. The AC/DC $\mathrm{PFC}$ stage is a rectifier followed by a boost converter. The PFC converter presents a varying voltage to the LLC stage. This increases the complexity of the optimization because LLC switching frequency must be varying in order to maintain output voltage regulation. The optimization method presented here deal with this issue. The PFC stage output voltage and its ripple is shown in Fig. 2. We take samples of the PFC output voltage in order to reduce the computation complexity of LLC operation.

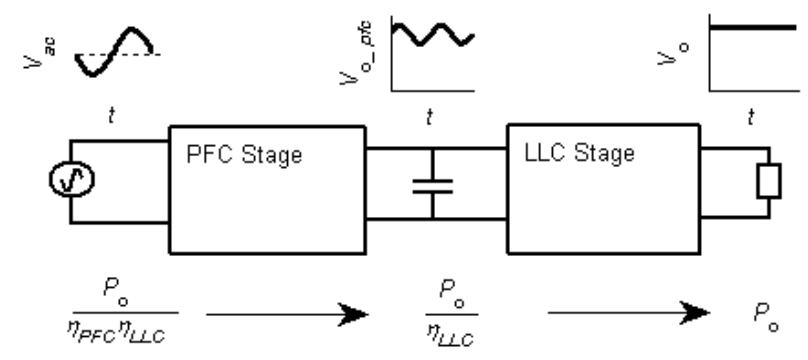

Fig. 1 A typical AC/DC DC/DC stage power converter.

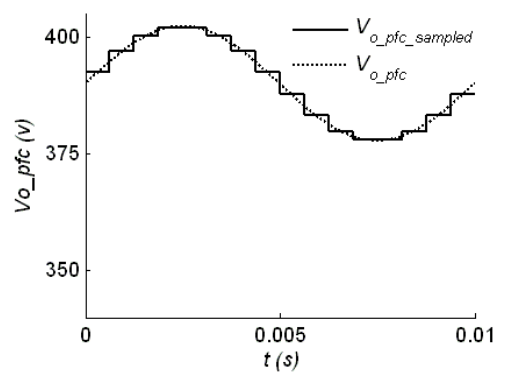

Fig. 2 Sampled PFC output voltage with ripple.

The peak-to-peak ripple PFC output voltage of is given by [4]:

$$
\left|\Delta V_{p f c-p p}\right|=\frac{P_{o}}{2 \pi f_{m} C_{p f c} V_{o-p f c}}
$$

where $V_{o-p f c}$ is the mean voltage of PFC output, $f_{m}$ is the line frequency, typically $50 \mathrm{~Hz}$ or $60 \mathrm{~Hz} . C_{p f c}$ is the value of 
the PFC output capacitor (input capacitor of LLC converter). $P_{o}$ is the output power.

\subsection{LLC series resonant converter}

The half-bridge LLC series resonant converter topology is shown in Fig. 3 Half-bridge LLC series resonant DC/DC converter.. The Equivalent circuits of LLC converter are shown in Fig. 4 Equivalent circuits of LLC converter..

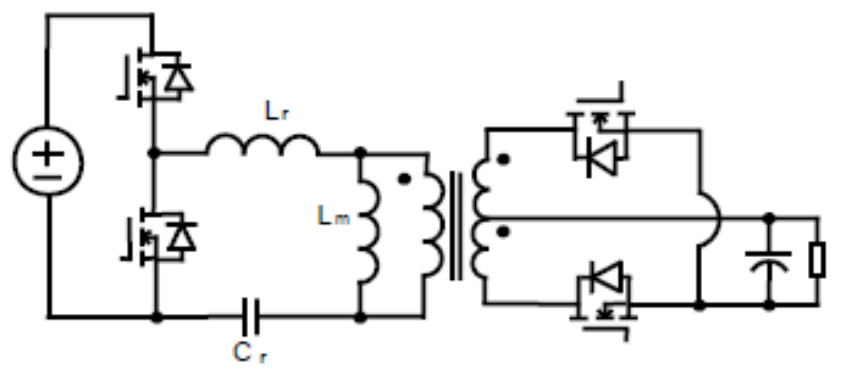

Fig. 3 Half-bridge LLC series resonant DC/DC converter.

A mode solver technique is proposed to compute the multiple-mode steady-state operation of the LLC converter. The proposed LLC mode solver serves as a function block in the main optimization procedure. The input variables of the LLC steady-state solver are the values of resonant parameters, such as $L_{r}, C_{r}, L_{m}$ and the required conversion gain $M$. Switching frequency at each sampled voltage $V_{o-p f c-s a m p l e d}$ is calculated in this stage. The state equations are also solved numerically in this stage and the output of this function block are vectors containing particular waveform information of current and voltage.

The mode solver starts with normalization for the sake of uniformity. $\omega_{0}$ and $\omega_{1}$ to denote the two resonant frequencies:

$$
\omega_{0}=\frac{1}{\sqrt{L_{r} C_{r}}}=2 \pi f_{r}, \omega_{1}=\frac{1}{\sqrt{\left(L_{r}+L_{m}\right) C_{r}}}
$$

Where $f_{r}$ is the resonance frequency.

The operation angle $\theta$ is given by

$$
\theta=\omega_{0} t
$$

$F$ is the ratio of the switching frequency and resonant frequency frequencies. The $\gamma$ is defined as a half period of switching cycle.

$$
F=\frac{f_{s}}{f_{r}}, \quad \gamma=\frac{\omega_{0}}{2 f_{s}}=\frac{\pi}{F}
$$

The conversion ration $M$ is defined as:

$$
M=\frac{V_{2}}{V_{1}}=\frac{2 n_{p} V_{\text {out }}}{n_{s} V_{\text {in }}}
$$

The operation angle $\theta$ is given by

$$
\theta=\omega_{0} t
$$

The conversion ration $M$ is defined as:

$$
M=\frac{V_{2}}{V_{1}}=\frac{2 n_{p} V_{\text {out }}}{n_{s} V_{\text {in }}}
$$

where V1 and V2 are input/output voltage of the equivalent circuits, $V_{\text {in }} V_{\text {out }}$ are the LLC input/output voltage, and $n_{p} n_{s}$ are the primary and secondary turns of transformer.

The normalization parameters are presented as followings:

$$
\begin{gathered}
V_{\text {base }}=V_{2}=\frac{n_{p} V_{\text {out }}}{n_{s}}, m_{2}=\frac{V_{2}}{V_{\text {base }}}=1 \\
m_{1}=\frac{1}{M}, Z_{\text {base }}=\sqrt{\frac{L_{r}}{C_{r}}}, I_{\text {base }}=\frac{V_{\text {base }}}{Z_{\text {base }}}
\end{gathered}
$$

Where $V_{\text {base }}$ is defined as the $V_{2}$ so that $m_{2}$ is normalized to unity, and $m_{l}$ is the normalized input voltage. The base impedance $Z_{\text {base }}$ and the base current $i_{\text {base }}$ are given by (10). The normalized voltage on resonant capacitor $m_{c}(\theta)$ and normalized current through resonant inductor $j_{L r}(\theta)$ are, respectively, given by

$$
m_{c}(\theta)=\frac{v_{c}\left(\frac{\theta}{\omega_{0}}\right)}{V_{\text {base }}}, j_{L r}(\theta)=\frac{i_{L r}\left(\frac{\theta}{\omega_{0}}\right)}{I_{\text {base }}}
$$

Similar expressions are applied to $m_{m}(\theta), m_{m 2}(\theta), m_{L r}(\theta)$, $j_{L m}(\theta)$ and $j_{\text {out }}$.

The ratio of two resonant inductance $\lambda$ and the ratio of two resonant frequencies $k_{l}$ are, respectively, given by (11). $r_{L}$ is the normalized load.

$$
\lambda=\frac{L_{r}}{L_{m}}, k_{1}=\frac{\omega_{1}}{\omega_{0}}, \quad r_{L}=\frac{n_{p}^{2} R_{L}}{n_{s}^{2} Z_{\text {base }}}
$$

where $R_{L}$ is the load resistance value.

The LLC resonant converter has several modes of operations. The discontinuous conduction mode below resonance (DCMB) and the continuous conduction mode above resonance (CCMA) are two main operation modes. And we only consider these two modes for simplicity.

\section{Discontinuous Conduction Mode below Resonance (DCMB)}

If $M>1$, we consider the LLC converter operates in Discontinuous Conduction Mode below Resonance. In DCMB, the LLC converter voltage conversion ratio $\mathrm{M}$ is larger than unity $(M>1)$. The equivalent circuit of DCMB mode in $[0, \alpha)$ is shown in Fig. 4(b). The state equations are given

$\theta \in[0, \alpha)$

$$
\left\{\begin{array}{l}
m_{c}(\theta)=\left[m_{c}(0)-\frac{1}{M}+1\right] \cos (\theta)+j_{L r}(0) \sin (\theta)+\frac{1}{M}-1 \\
m_{m}(\theta)=1 \\
j_{L r}(\theta)=\left[-m_{c}(0)+\frac{1}{M}-1\right] \sin (\theta)+j_{L r}(0) \cos (\theta) \\
j_{L m}(\theta)=j_{L m}(0)+\lambda \theta
\end{array}\right.
$$



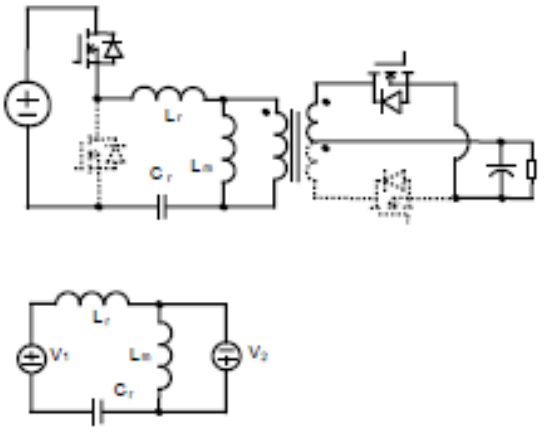

(a)
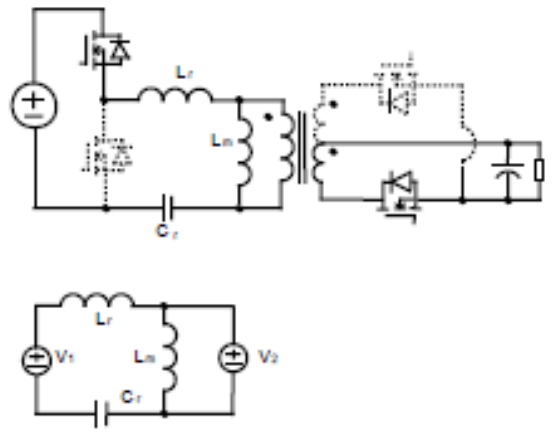

(b)
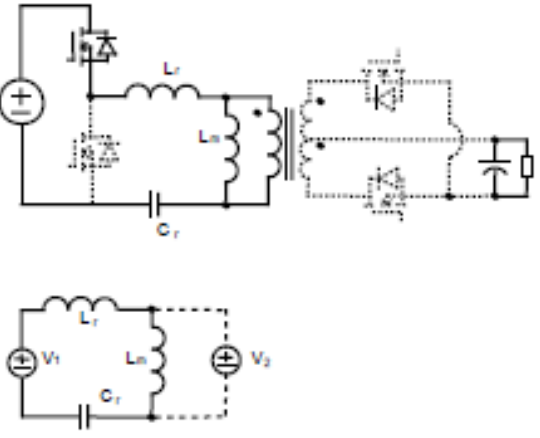

(c)

current harmonics are calculated by fast Fourier transform, as shown in Fig. 6.

The equivalent circuit of DCMB mode in $[\alpha, \gamma)$ is shown in Fig. 4(c). The state equations are given by

$\theta \in[\alpha, \gamma)$

$$
\left\{\begin{array}{l}
m_{c}(\theta)=\left[m_{c}(\alpha)-\frac{1}{M}\right] \cos \left[k_{1}(\theta-\alpha)\right]+\frac{j_{L r}(\alpha)}{k_{1}} \sin \left[k_{1}(\theta-\alpha)\right]+\frac{1}{M} \\
m_{m}(\theta)=\left\{\left[-m_{c}(\alpha)+\frac{1}{M}\right] \cos \left[k_{1}(\theta-\alpha)\right]-\frac{j_{L r}(\alpha)}{k_{1}} \sin \left[k_{1}((\theta-\alpha)]\right\} /(1+\lambda)\right. \\
j_{L r}(\theta)=\left[-m_{c}(\alpha)+\frac{1}{M}\right] k_{1} \sin \left[k_{1}(\theta-\alpha)\right]+j_{L r}(\alpha) \cos \left[k_{1}(\theta-\alpha)\right] \\
j_{L m}(\theta)=j_{L r}(\theta)
\end{array}\right.
$$

The average output current $j_{\text {out }}$ is given by

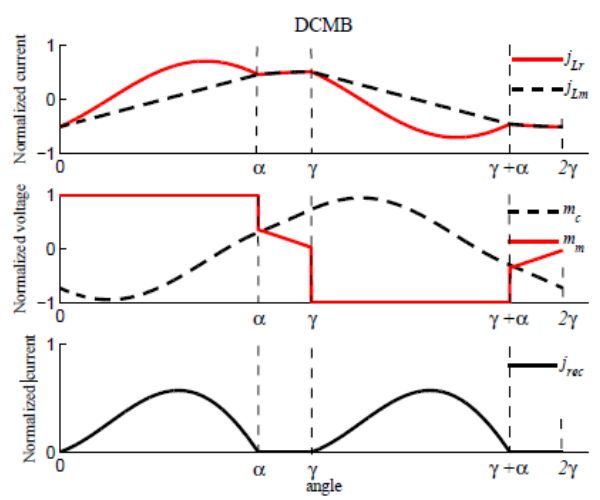

$$
\begin{aligned}
j_{\text {out }} & =\frac{1}{\gamma} \int_{0}^{\gamma}\left|j_{L r}(\theta)-j_{L m}(\theta)\right| d \theta \\
& =\frac{1}{\gamma} \int_{0}^{\alpha}\left[j_{L r}(\theta)-j_{L m}(\theta)\right] d \theta \\
& =\frac{1}{\gamma}\left\{\left[-m_{c}(0)+\frac{1}{M}-1\right](1-\cos \alpha)+j_{L r}(0) \sin \alpha-j_{L r}(0) \alpha-\frac{1}{2} \lambda \alpha^{2}\right\}
\end{aligned}
$$

The steady-state solution in DCMB $\left[j_{L r}(0), m_{c}(0), \alpha, M\right]$ can be solved by:

$$
\left\{\begin{array}{l}
m_{c}(0)+m_{c}(\gamma)=0 \\
j_{L r}(0)+j_{L r}(\gamma)=0 \\
j_{L r}(\alpha)-j_{L m}(\alpha)=0 \\
j_{\text {out }} r_{L}-1=0
\end{array}\right.
$$

These four equations become the basic of the solver, which adequately describe the waveforms of the resonant operation. Since these unknowns do not have the analytical closed-form solution, the equations are solved by MATLAB function fsolve( $\mathrm{x}$ ) which is a numerical based search function.

If $\mathrm{M}<1$, we consider the LLC converter operate in CCMA mode. The state equations of CCMA are similar to that of DCMB. Typical waveforms of DCMB and CCMA operations are presented in Fig. 5.

The current waveforms of LLC converter are determined by the operation mode and calculated by the proposed mode solver. Current harmonics are calculated to predict losses. A numerical method is used to sample a switching cycle. The

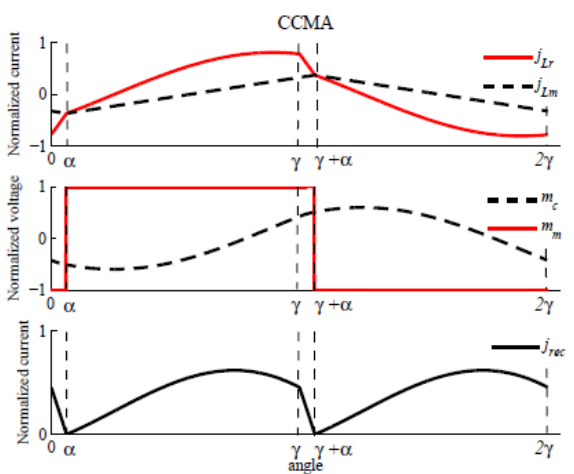

Fig. 5 LLC operation modes DCMB and CCMA
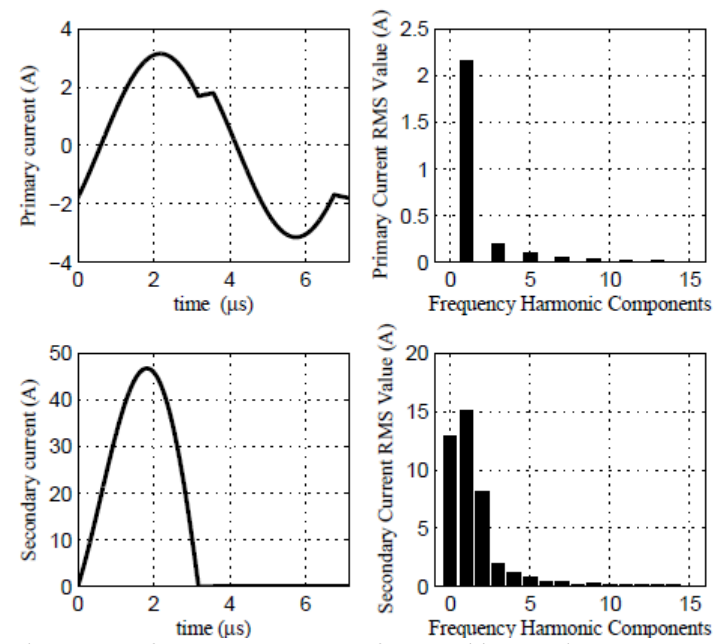

Fig. 6 Transformer current waveform and harmonic components 


\section{Converter loss models}

Converter loss models are reviewed in this section. The main components are primary MOSFETs, synchronous rectifiers, transformer, resonant inductor, resonant capacitor, input/output capacitors.

\section{Primary MOSFETs:}

The conduction loss of primary MOSFETs are given by

$$
P_{c d-p r i}=I_{R M S-p r i}^{2} R_{d s-p r i}
$$

Where $I_{R M S-p r i}$ is root mean square value of primary current, $R_{d s-p r i}$ is the "on" resistance of primary MOSFETs.

Since zero voltage turn-on can be achieved in LLC converter, we assume there is no switching loss. Please note this assumption is under the condition that turn-off current of primary MOSFETs is low.

\section{Synchronous rectifiers:}

Synchronous rectification (SR) is implemented at the secondary side to achieve high efficiency at the low-voltage high-current output condition. The current driven synchronous rectifier driving scheme is implemented. We assume the SR works under a timing scheme that current does not flow through synchronous rectifier body diode. The major losses for the synchronous rectifier are the conduction loss, turn-off switching loss and the gate drive loss. Turn-off switching loss is the energy stored in the stray inductance and being dissipated by the circuit. The simplified model for the turn-off loss and the gate driving loss of SR are denoted as $P_{S W-S R}$ and $P_{g-S R}$ respectively. The conduction loss of SRs is denoted as $P_{c d-S R}$.

$$
\begin{aligned}
& P_{s w-S R}=\frac{n_{s} V_{i n} Q_{o s S_{-} S R} f_{s}}{2 n_{p}} \\
& P_{g-S R}=Q_{g-S R} V_{g-S R} f_{s} \\
& P_{c d-S R}=I_{R M S-\sec }^{2} R_{d s-S R}
\end{aligned}
$$

\section{Magnetic components:}

Transformer and resonant inductor are two magnetic components in LLC converter. The AC copper loss is worked out as below.

The skin depth of the $n^{\text {th }}$ harmonics frequency is given by

$$
\delta(n)=\sqrt{\frac{2 \rho_{c u}}{2 \pi n f_{s} \mu_{0}}}
$$

The AC-to-DC resistance ratio $F_{R}$ at $n_{t h}$ harmonic frequency is calculated by Dowell's equation [5] given by

$$
\begin{aligned}
F_{R}(n, p, X)= & X \frac{e^{2 X}-e^{-2 X}+2 \sin (2 X)}{e^{2 X}+e^{-2 X}-2 \cos (2 X)} \\
& +2 X \frac{p^{2}-1}{3} \frac{e^{X}-e^{-X}-2 \sin (2 X)}{e^{X}+e^{-X}+2 \cos (2 X)}
\end{aligned}
$$

We have: $F_{R-p r i}(n)=F_{R}(n, p, X)$ is for primary round wire with $p=n_{\text {layer }}$ and $X=\frac{\sqrt{\pi} d_{A W G}}{2 \delta(n)} . F_{R \text {-sec }}(n)=F_{R}(n, p, X)$ is for secondary foils with $p=\frac{n_{s}}{2} \quad X=\frac{h_{f o i l}}{\delta(n)}$
$F_{R-L r}(n)=F_{R}(n, p, X)$ is for resonant inductor round wire with $p=\frac{n_{s}}{2}$ and $X=\frac{h_{\text {foil }}}{\delta(n)}$.

The AC copper loss at each harmonic frequency is calculated by summing the losses from DC to $32^{\text {th }}$ harmonics. The primary side of transformer are given by

$$
P_{c u-X F-p r i}=R_{X F-p r i} \sum_{n=0}^{32} F_{R-p r i} I_{n-p r i}^{2}
$$

Where $R_{X F-p r i}$ is the DC resistance of transformer primary side winding calculated by geometry, $I_{n-p r i}$ is the $n^{\text {th }}$ harmonic current at the primary side calculated by Fast Fourier Transform (FFT). Similar manner can be applied to the calculation of secondary foils copper loss and resonant inductor copper loss.

\section{Capacitors:}

The resonant capacitor in series with the power path carries high RMS current and high voltage. A low loss capacitor is used to achieve high efficiency and low temperature. Metalized polypropylene capacitor is selected because of its low dissipation factor and low cost. The dissipation factor $D_{F}$ of polypropylene capacitor (or loss angle $\tan \delta$ ) increases with the increasing of frequency up to $10 \mathrm{MHz}$. We assume $D_{F}$ increase linearly with the frequency given by (23).

$$
\begin{aligned}
D_{F} & =a_{D F} f+b_{D F} \\
R_{C r} & =\frac{D_{F}}{2 \pi f C_{r}} \\
P_{C r} & =I_{P M S-p r i}^{2} R_{C r}
\end{aligned}
$$

where $a_{D F}$ and $b_{D F}$ are constant, $f$ is the frequency, $R_{C r}$ is the equivalent series resistance (ESR) of resonant capacitor. $P_{C r}$ is the loss of resonant capacitor.

\section{Optimization procedures}

An optimization procedure is presented in this section. The optimization program in this paper is developed under MATLAB environment. The LLC efficiency optimization involves non-linear, constrained, continuous optimization problems. The fmincon(x) function of MATLAB optimization toolbox is applied as the optimizer to solve such problems. The "activeset" algorithm is used in the fmincon(x) function. Detailed optimization procedures can be found in []. The aim of the optimization is to minimize the loss at a certain loading condition. The flow chart of optimization procedure is presented in

Fig. 7. The characteristics of the power components are discrete, such as the primary MOSFETs, transformer core and bobbin size. The continuous optimization methods cannot handle such discrete values, so we pre-select the discrete components at the discrete component selection stage. In the continuous optimization stage, the discrete components and their related parameters are fixed. Let $x$ denote a vector containing all the design variables, such as primary turns, secondary turns, value of $L_{r}, L_{m}$ and $C_{r}$ etc.. 


$$
\begin{aligned}
x= & {\left[n_{p}, n_{s}, L_{m}, C_{r}, L_{r}, d_{A W G},\right.} \\
& \left.n_{\text {layer }}, h_{\text {foil }}, n_{L r}, d_{A W G-L r}, n_{\text {Lr-layer }}\right]
\end{aligned}
$$

where $n_{p} n_{s}$ are numbers of primary turns, $L_{m} C_{r} L_{r}$ are values of resonant tank, $d_{A W G}$ is the diameter of the transformer wire, $n_{\text {layer }}$ is the number of layer of transformer, $h_{\text {foil }}$ is the thickness of the transformer secondary foils, $n_{L r}$ is the number of turns of resonant inductor, $d_{A W G-L r}$ is the diameter of wire of the resonant inductor, and $n_{\text {Lr-layer }}$ is the number of layers of the resonant inductor.

The mean loss at each LLC input voltage is given by

$$
P_{\text {loss-mean }}(x)=\frac{1}{n_{\text {in }}} \sum_{i=1}^{n_{\text {in }}} P_{\text {loss }}(x, i)
$$

Where $n_{\text {in }}$ is the number of sampled LLC input voltage. The optimization problem is to minimize the mean loss $P_{\text {loss-mean }}(x)$ subject to constraints set $\Omega$, given by

$$
\min _{x \in \Omega} P_{\text {loss-mean }}(x)
$$

Where $\Omega$ is given by

$$
\begin{gathered}
\Omega=\left\{x \mid b_{x l} \leq \leq x \leq \leq b_{x u}, 0.3-\Delta B_{m-L r} \geq 0\right. \\
\left.0.3-\Delta B_{m-X F} \geq 0\right\}
\end{gathered}
$$

The lower bound vector $b_{x l}$ and the upper bound vector $b_{x u}$ of design variables give the searching range, where the expression " $x \geq \geq b_{x l}$ " denotes " $x-b_{x l}$ " to be a vector with non-

\begin{tabular}{|c|c|c|c|c|}
\hline $\begin{array}{c}\text { Design } \\
\text { Variables }\end{array}$ & $\begin{array}{l}\text { Lower } \\
\text { bound }\end{array}$ & $\begin{array}{l}\text { Upper } \\
\text { bound }\end{array}$ & $\begin{array}{l}\text { Optima } \\
\text { Results }\end{array}$ & $\begin{array}{c}\text { Stopping } \\
\text { at boundary }\end{array}$ \\
\hline$n_{p}$ & 10 & 60 & 32.8 & No \\
\hline$n_{s}$ & 1 & 3 & 2 & No \\
\hline$L_{m}(\mu H)$ & 100 & 300 & 300 & Yes \\
\hline$C_{r}(n F)$ & 10 & 100 & 55 & No \\
\hline$L_{r}(\mu H)$ & 22 & 90 & 22 & Yes \\
\hline $\begin{array}{c}d_{X F-A W G} \\
(\mathrm{~mm})\end{array}$ & 0.1 & 0.5 & 0.1 & Yes \\
\hline$n_{X F-\text { layer }}$ & 1 & 20 & 4.8 & No \\
\hline $\begin{array}{c}d_{L r-A W G} \\
(\mathrm{~mm})\end{array}$ & 0.1 & 0.2 & 0.1 & No \\
\hline$n_{L r}$ & 1 & 50 & 11 & No \\
\hline$h_{\text {foil }}(\mathrm{mm})$ & 0.1 & 0.2 & 0.1 & Yes \\
\hline$n_{\text {Lr-laver }}$ & 1 & 20 & 5 & No \\
\hline
\end{tabular}
negative entries. " $0.3-\Delta B_{m-L r} \geq 0$ " and " $0.3-\Delta B_{m-X F} \geq 0$ " denote that the resonant inductor and the transformer do not saturate ( 0.3 is assumed to be the ferrite flux saturation level).

The optimization program searches the optimal result that fulfils the constraints set.

Table: 1 Optimized results

\section{Optimization and experimental results}

\subsection{Optimization results}

The optimization program aims to optimize a $12 \mathrm{~V}$ output voltage and $25 \mathrm{~A}$ output current LLC resonant converter. The optimized design variables and lower/upper bounds are presented in Table 1. The lower/upper bounds are predefined. It can be seen that some of the design variables converge to their boundaries. These boundaries are limited by physical factors such as size. This table also indicates those boundaries that can be improved to have even higher efficiency.

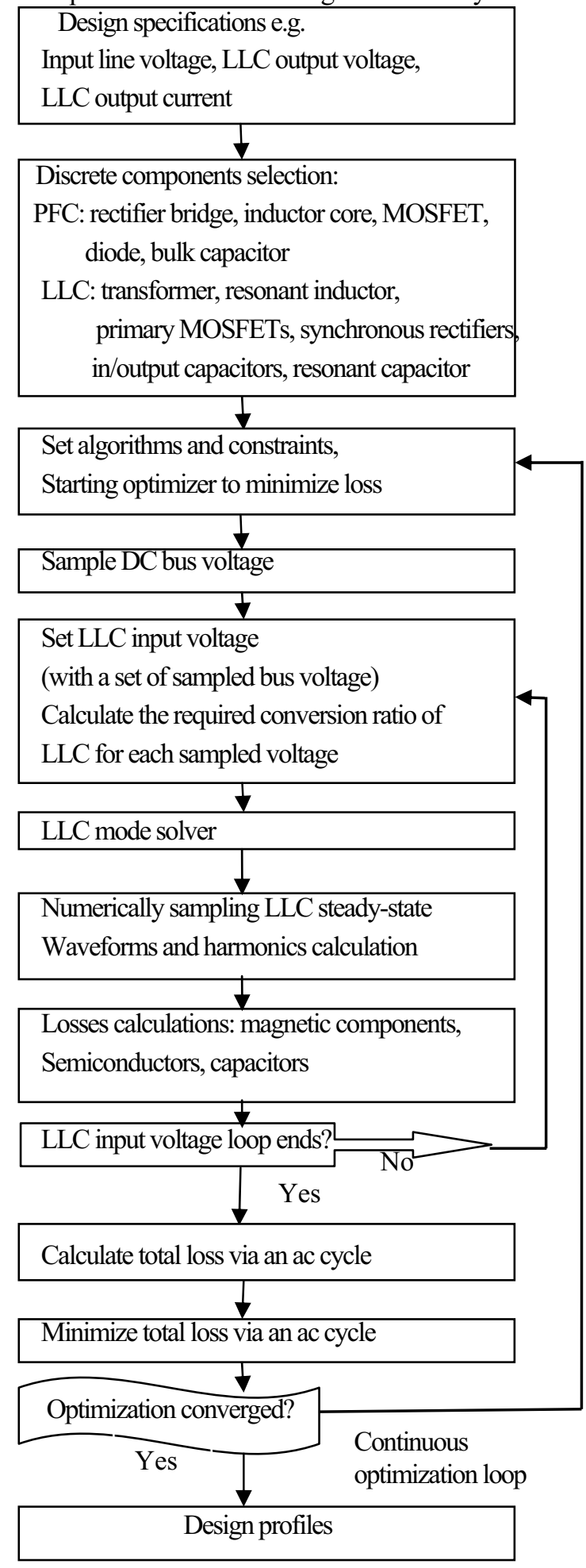

Fig. 7 Main optimization procedures

The optimized LLC converter efficiency is calculated to be $96.6 \%$, where the calculated efficiency is (output power)/[(all losses)+(output power)]. 


\begin{tabular}{|l|l|l|}
\hline \multicolumn{3}{|c|}{ COMPONENTS LIST } \\
\hline $\begin{array}{l}\text { LLC Primary } \\
\text { MOSFETs }\end{array}$ & IPP50R140CP & \\
\hline Synchronous rectifier & BSC016N04LS3 & Current driven SRs \\
\hline LLC transformer & $\begin{array}{l}\text { Turn ratio 33:2 } \\
\text { ETD44/22/15 } \\
\text { 3C90 }\end{array}$ & $\begin{array}{l}\text { Primary: AWG40*50 } \\
\text { Secondary: } 0.2 \mathrm{~mm} \text { foils } \\
\text { Leakage: } 8 \mu \mathrm{H}\end{array}$ \\
\hline Resonant inductor & $\begin{array}{l}17 \mu H \text { PQ20/16 } \\
\text { 3C96 }\end{array}$ & AWG40*60 \\
\hline Resonant capacitor & $47 n F 1000 \mathrm{~V}$ & $\begin{array}{l}\text { Metalized polypropylene } \\
1.72 \mathrm{KP} .047 / 201000 \mathrm{~V}\end{array}$ \\
\hline $\begin{array}{l}\text { LLC Input Capacitor } \\
\text { (PFC output Capacitor) }\end{array}$ & $200 \mu F$ 450V & RUBYCON KXW \\
\hline PFC Choke & $600 \mu H$, & $\begin{array}{l}\text { AWG38*30*2 } \\
70 \text { turns }\end{array}$ \\
\hline PFC MOSFET & IPP60R299CP & \\
\hline PFC Diode & IDH04SG60C & \\
\hline $\begin{array}{l}\text { PFC Controller: NCP1654 } \\
\text { LLC controller: NCP1397 }\end{array}$ & \\
\hline
\end{tabular}

Table 2: Components list

\subsection{Experimental results}

A prototype PFC+LLC converter is built to verify the optimization results as shown in Fig. 8. The components list is shown in Table 2. The measured efficiency at $230 \mathrm{Vac}$ is presented in Fig. 9. The full load efficiency at 230Vac is $94.6 \%$. The waveform of LLC input voltage ripple is shown in Ch1 of Fig. 10, the voltage after bridge rectifier is shown in $\mathrm{Ch} 2$ and the LLC resonant current is shown in $\mathrm{Ch} 4$.

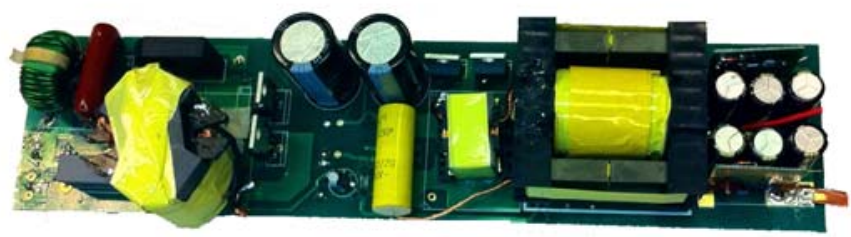

Fig. 8 Prototype PFC and LLC converter.

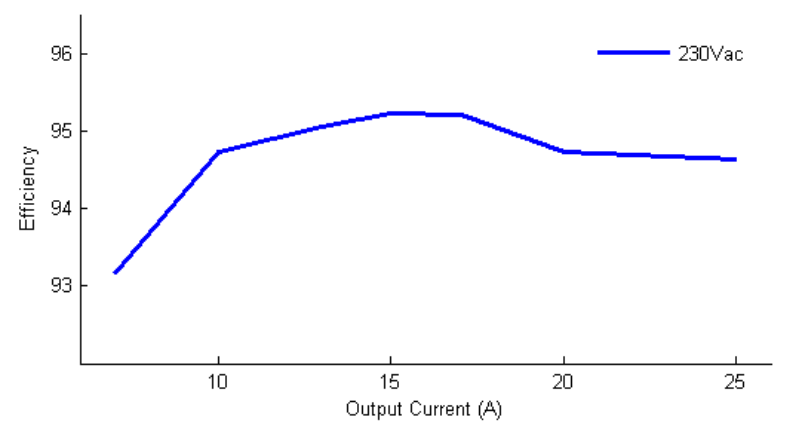

Fig. 9 Measured efficiency at 230Vac

\section{Conclusions}

A systematic optimization procedure is presented in this paper to optimize LLC series resonant converter efficiency where the effect of LLC input voltage variation cased by power factor correction circuit is considered. A mode solver technique is proposed to handle LLC converter steady-state solutions under different input voltage conditions. Loss models are reviewed. A prototype $300 \mathrm{~W} 12 \mathrm{~V}$ output LLC converter is built using the optimization results and high efficiency is obtained.

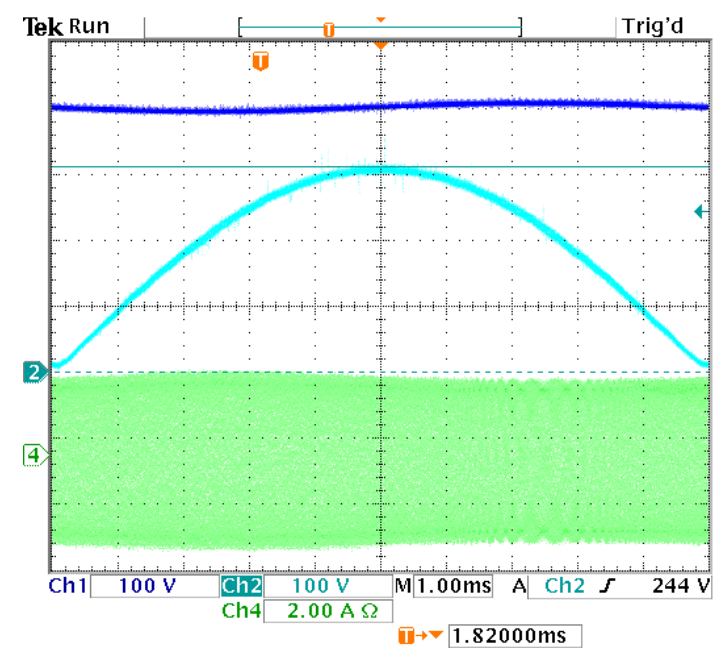

Fig. 10 converter waveforms

\section{References}

[1] S. Balachandran and F. C. Lee, "Algorithms for power converter design optimization,". IEEE Trans. Aerosp Electron. Syst., vol. AES-17, no. 3,pp. 422-432, May 1981.

[2] T. C. Neugebauer and D. J. Perreault, "Computer-Aided optimization of DC/DC converters for automotive applications," IEEE Trans. Power Electron., vol. 18, no. 3, pp.775-783, May 2003.

[3] U. Badstuebner, J. Biela and J. W. Kolar, "Design of an 99\%-efficient, $5 \mathrm{~kW}$, phase-shift PWM DC-DC converter for telecom applications, "In Proc. IEEE Applied Power Electronics Conf., 2010, pp. 773-780.

[4] C.K. Tse, "Circuit Theory of Power Factor Correction in Switching onverters." International Journal of Circuit Theory and Applications, vol. 1, no. 2, pp. 157-198, March 2003.

[5] P. L. Dowell, "Effect of eddy currents in transformer windings," IEE Proc., vol. 113, no. 8, pp. 1387-1394, Aug. 1966. 